\title{
Erratum: Fluidity of Hydration Layers Nanoconfined between Mica Surfaces [Phys. Rev. Lett. 94, 026101 (2005)]
}

\author{
Yongsheng Leng and Peter T. Cummings \\ (Received 28 January 2005; published 17 February 2005)
}

DOI: 10.1103/PhysRevLett.94.069902

PACS numbers: 68.08.-p, 61.20.-p, 81.40.Pq, 99.10.Cd

The last sentence on the first page of the above manuscript (beginning "Our detailed structure calculations ...") is missing a reference to the $\mathrm{x}$-ray reflectivity experiment of Cheng et al. [1]. Likewise, the following sentence (beginning "A similar density oscillation ...") is missing a reference to the Monte Carlo simulations of Park and Sposito [2]. These references were inadvertently deleted during the process of reducing the manuscript to fit within the length constraint of Physical Review Letters.

[1] L. Cheng et al., Phys. Rev. Lett. 87, 156103 (2001).

[2] S. H. Park and G. Sposito, Phys. Rev. Lett. 89, 85501 (2002). 